\title{
Acurácia do teste de força muscular da cinesiologia aplicada para disfunção sacroilíaca*
}

\section{Accuracy of the Applied Kinesiology Muscle Strength Test for Sacroiliac Dysfunction}

\author{
Jaisson Agne Estrázulas ${ }^{10}$ Lauro Sinval Bueno ${ }^{2}$ Luana Rocha de Oliveira Lombardi²
} Jansen Atier Estrázulas ${ }^{3}$ Tiótrefis Gomes Fernandes ${ }^{1}$ Juliana Albuquerque Baltar ${ }^{1,4}$

${ }^{1}$ Faculdade de Educação Física e Fisioterapia, Universidade Federal do Amazonas (UFAM), Manaus, Brasil

2 Bacharel em Fisioterapia, Manaus (AM), Brasil

3 Universidade do Estado do Amazonas (UEA), Manaus, Brasil

4 Programa de Pós-graduação em Medicina, Universidade Federal de São Paulo, SP, Brasil

Rev Bras Ortop 2020;55(3):293-297.

\author{
Endereço para correspondência Jaisson Agne Estrázulas, ESP, \\ Universidade Federal do Amazonas, Avenida General Rodrigo Octavio \\ Jordão Ramos 1.200, Coroado I, Manaus, AM, 69067-005, Brasil \\ (e-mail: jaissonagne@hotmail.com).
}

\section{Resumo}

\section{Palavras-chave}

- articulação sacroilíaca

- cinesiologia aplicada

- sensibilidade e especificidade
Objetivo Investigar a acurácia do teste de força muscular da cinesiologia aplicada para disfunção sacroilíaca, comparando-o com quatro testes ortopédicos validados.

Métodos Trata-se de uma pesquisa transversal de acurácia desenvolvida em uma clínica particular da cidade de Manaus no mês de fevereiro de 2017. A amostra teve 20 indivíduos, com mediana de idade 33,5 anos. Foram aplicados quatro testes: distração, thigh thrust, compressão e thrust sacral, e o diagnóstico foi confirmado quando três destes testes deram positivo. Logo após, foi aplicado o teste da cinesiologia aplicada para o músculo piriforme.

Resultados A prevalência de disfunção da articulação sacroilíaca observada foi de 45\%, tendo o teste thigh thrust alta especificidade, e o teste thrust sacral, alta sensibilidade. 0 teste da cinesiologia aplicada obteve bons resultados (sensibilidade: 0,89 ; especificidade: 0,82; valor preditivo positivo: 0,80 ; valor preditivo negativo: 0,82 ; acurácia: 0,85 ; área abaixo da curva de característica de operação do receptor [COR]: 0,85 ).

Conclusão $\mathrm{O}$ teste de força muscular da cinesiologia aplicada, de grande viabilidade clínica, mostrou ter boa acurácia no diagnóstico da disfunção da articulação sacroilíaca, e maior poder discriminatório da disfunção existente, em comparação aos demais testes.

Trabalho desenvolvido na Universidade Federal do Amazonas,

Manaus, AM, Brasil.

recebido

25 de Setembro de 2018

aceito

22 de Janeiro de 2019
DOI https://doi.org/

10.1055/s-0039-1700832. ISSN 0102-3616.
Copyright $\odot 2020$ by Sociedade Brasileira License terms de Ortopedia e Traumatologia. Published by Thieme Revinter Publicações Ltda, Rio de Janeiro, Brazil 


\author{
Keywords \\ - sacroiliac joint \\ - applied kinesiology \\ - sensitivity and \\ specificity
}

tests were positive. Soon after, the applied kinesiology test was applied to the piriformis muscle.

Results The prevalence of sacroiliac joint dysfunction was of $45 \%$; the thigh thrust test had the highest specificity, and the sacral thrust test had the highest sensitivity. The applied kinesiology test obtained good results (sensitivity: 0.89 ; specificity: 0.82 ; positive predictive value: 0.80 ; negative predictive value: 0.82 ; accuracy: 0.85 ; and area under the receiver operating characteristic [ROC] curve: 0.85 ).

Conclusion The applied kinesiology muscle strength test, which has great clinical feasibility, showed good accuracy in diagnosing sacroiliac joint dysfunction and greater discriminatory power for the existing dysfunction in comparison to the other tests.

\section{Introdução}

A coluna lombar é alvo de constantes quadros de dor, conhecidos como lombalgia, com prevalência que varia de $38,9 \%$ a $70 \%$ ao longo da vida, ${ }^{1,2}$ sendo considerado um problema crítico de saúde pública. ${ }^{3}$ A articulação sacroilíaca é aceita como uma fonte potencial de lombalgia e/ou dor na região glútea num percentual que varia entre $10 \%$ e $27 \%$ dos indivíduos afetados. ${ }^{4-6}$ A disfunção da articulação sacroilíaca (DASI) ocorre devido a um mau alinhamento ou movimento anormal dos ossos do ilíaco e do sacro, gerando dor na articulação ou em torno dela. ${ }^{4}$

O teste diagnóstico tido como padrão ouro para a DASI é o bloqueio articular por meio de injeção de anestésico intraarticular, guiado por fluoroscopia. Porém, este é um procedimento invasivo, que expõe o indivíduo a radiação e apresenta alto grau de dificuldade na aplicação. ${ }^{7}$ Os testes ortopédicos compressivos, que provocam a dor, apresentam boa validade para diagnosticar uma disfunção sacroilíaca, ${ }^{8-10}$ sendo necessário aplicar quatro testes e obter resultado positivo em pelo menos três. ${ }^{7,10-12}$

A cinesiologia aplicada (CA) foi desenvolvida na década de 1960 por George Goodheart, quiroprático americano, que associou a função muscular com o sistema craniossacral, os meridianos de energia, e os fatores hormonais, nutricionais, e emocionais, além da reflexologia, na qual cada músculo estaria relacionado a órgãos específicos do corpo. ${ }^{13,14}$ Este sistema de avaliação utiliza métodos de diagnósticos específicos, como o teste muscular manual, que avalia a mudança na resposta neuromuscular para determinar como e onde o corpo está desequilibrado, para que possa ser corrigido adequadamente. Esse método utiliza estímulos, também chamados de desafios, para comparar a reação muscular pré-teste e pós-teste, sendo um desafio considerado positivo quando há a alteração de reação de um músculo, ${ }^{13,15}$ ou seja, quando uma área desequilibrada é submetida a um estímulo, o sistema nervoso, que já se encontra sobrecarregado, faz com que o músculo, anteriormente normorreativo, fique não reativo temporariamente.

O International College of Applied Kinesiology (ICAK) vem buscando testar a eficácia de sua técnica para facilitar tanto o diagnóstico quanto o tratamento ${ }^{13}$ de diversas disfunções como a lombalgia. Os poucos estudos encontrados a respeito da CA tendem a utilizar os estímulos químicos, por meio de alguma substância nociva ao ser humano, e os estímulos emocionais para buscar a comprovação da eficácia do método. ${ }^{16-19}$ Contudo, a literatura carece de estudos a respeito da acurácia do desafio mecânico, utilizando a aplicação de seus conceitos em pacientes com diferentes tipos de disfunções musculoesqueléticas. Em face do exposto, o objetivo deste trabalho foi investigar a acurácia do teste de força muscular da CA para disfunção da articulação sacroilíaca, por meio do desafio mecânico, em comparação com outros testes ortopédicos já validados.

\section{Metodologia}

Foi realizado um estudo de acurácia, descritivo, transversal, no mês de fevereiro de 2017. A amostra não probabilística, por conveniência, foi composta por 20 indivíduos. Foram incluídos os indivíduos que no dia da coleta estiveram presentes na clínica com suspeita de DASI, tendo eles assinado o termo de consentimento livre e esclarecido. Como critérios de exclusão, utilizaram-se a presença de prótese de qualquer natureza na região do quadril, malformação congênita, e pacientes que não tinham a cognição preservada para compreender a dinâmica do teste. O estudo foi aprovado pelo Comitê de Ética em Pesquisa em Seres Humanos da nossa instituição, sob CAAE $\mathrm{n}^{\circ}$ 62554916.7.0000.5020 e parecer $\mathrm{n}^{\circ}$ 1.901.399.

Os sujeitos foram avaliados por três profissionais distintos. O primeiro avaliador realizou uma anamnese, na qual foram registrados dados pessoais e a investigação de dor na região da articulação sacroilíaca. $O$ segundo avaliador aplicou os quatro testes ortopédicos: distração, thigh thrust, compressão e thrust sacral, ${ }^{4,20}$ escolhidos por apresentarem altos valores de sensibilidade e especificidade. ${ }^{4,7,8,10,20-22}$ Foi considerado um diagnóstico positivo para DASI quando pelo menos três dos quatro testes aplicados deram positivo., 10-12

Logo em seguida, o terceiro avaliador, fisioterapeuta com formação em osteopatia e nos métodos da CA, aplicou o teste manual de força da CA para DASI, utilizando o desafio mecânico para o piriforme (músculo com origem nesta articulação escolhido para o estudo). A posição inicial para o teste muscular foi a que colocou o músculo a ser testado na maior vantagem, com os agentes sinérgicos em desvantagem, ${ }^{23,24}$ 
conforme descrito a seguir. O teste funciona da seguinte forma: ${ }^{24}$

Fase 1-Verificação da musculatura pré-teste:

- $1^{\circ}$ passo: com o sujeito em decúbito ventral, o avaliador solicita que faça uma flexão de joelho unilateral de $90^{\circ}$ e uma leve rotação externa e abdução de quadril, entre $5^{\circ}$ e $10^{\circ}$;

- $2^{\circ}$ passo: o avaliador posiciona a mão cefálica na porção lateral do joelho para impedir a abdução de quadril, e a mão caudal, na região interna mais distal da perna flexionada, para resistir à rotação externa de quadril;

- $3^{\circ}$ passo: solicita-se ao paciente uma rotação externa e uma abdução da coxa, contra a resistência manual do avaliador, que impedirá o movimento, resultando em uma contração isométrica;

- $4^{\circ}$ passo: o avaliador irá resistir até que não detecte mais aumento de força contra sua mão. Neste ponto, uma força pequena adicional será exercida pelo avaliador em uma tangente ao arco criado pela parte do corpo a ser testado, ou seja, tentando rodar internamente a coxa;

- $5^{\circ}$ passo: o avaliador verifica a resposta do músculo testado, podendo ser um músculo que resistiu à pressão, normorreativo, ou que não resistiu à pressão, não reativo.

Fase 2-Realização do desafio mecânico:

- Para testar uma possível disfunção, o avaliador realiza a mobilização passiva no sentido da correção dela, sustenta por poucos segundos e, em seguida, solta de forma abrupta, causando assim uma exacerbação da condição da possível lesão. No caso do estudo em questão, foi feita manualmente uma das seguintes situações, visando diagnosticar possível disfunção: base direita do sacro mobilizada no sentido anteroinferior; base esquerda do sacro mobilizada no sentido anteroinferior; ilíaco direito mobilizado no sentido posterossuperior; ilíaco direito mobilizado no sentido anteroinferior; ilíaco esquerdo mobilizado no sentido posterossuperior; ilíaco esquerdo mobilizado no sentido anteroinferior.

Fase 3-Verificação da musculatura pós-teste:

- Após cada desafio descrito na fase 2, o avaliador irá refazer os passos da fase 1 e verificar se houve um desafio negativo (músculo mantém a mesma reação da primeira fase); ou se há um desafio positivo (músculo apresenta modificação da reação da primeira fase).

Fase 4-Conclusão do teste:

- Conclusão 1: caso o avaliador perceba que não há diferença nas reações antes e depois em qualquer um dos desafios descritos na fase 2, o teste é considerado negativo para DASI;

- Conclusão 2: caso o avaliador perceba que há diferença nas reações antes e depois em qualquer um dos desafios descritos na fase 2, o teste é considerado positivo para DASI, sendo possível identificar com precisão a disfunção que está levando ao quadro álgico e, assim, orientar o tratamento.
Tabela 1 Distribuição da amostra segundo sexo, idade e sintomatologia dolorosa

\begin{tabular}{|c|c|c|c|}
\hline Variável & $\begin{array}{l}\text { Amostra } \\
\text { total } \\
(n=20)\end{array}$ & $\begin{array}{l}\text { DASI + } \\
(n=9)\end{array}$ & $\begin{array}{l}\text { DASI - } \\
(n=11)\end{array}$ \\
\hline \multicolumn{4}{|l|}{ Gênero $(n)$} \\
\hline Feminino & $30 \%(n=6)$ & $50 \%(n=3)$ & $50 \%(n=3)$ \\
\hline Masculino & $\begin{array}{l}70 \% \\
(n=14)\end{array}$ & $\begin{array}{l}42,9 \% \\
(n=6)\end{array}$ & $\begin{array}{l}57,1 \% \\
(n=8)\end{array}$ \\
\hline Idade* (anos) & $\begin{array}{l}33,5 \\
(26,8-43,0)\end{array}$ & $\begin{array}{l}36 \\
(27,8-43,0)\end{array}$ & $\begin{array}{l}33,5 \\
(26,8-43,0)\end{array}$ \\
\hline \multicolumn{4}{|l|}{$\begin{array}{l}\text { Sintomatologia } \\
\text { de dor }\end{array}$} \\
\hline Sim & $\begin{array}{l}55 \% \\
(n=11)\end{array}$ & $\begin{array}{l}77,8 \% \\
(n=7)\end{array}$ & $\begin{array}{l}36,4 \% \\
(n=4)\end{array}$ \\
\hline Não & $\begin{array}{l}45 \% \\
(n=9)\end{array}$ & $\begin{array}{l}22,2 \% \\
(n=2)\end{array}$ & $\begin{array}{l}63,6 \% \\
(n=7)\end{array}$ \\
\hline
\end{tabular}

Abreviatura: DASI, disfunção da articulação sacroilíaca.

Nota: *Resultado expresso em mediana e intervalo interquartil (IIQ).

\section{Análise dos Dados}

Foi feito estudo descritivo das variáveis, segundo sua natureza e distribuição. Com o programa Statistical Package for the Social Sciences (SPSS, IBM Corp., Armonk, NY, EUA), versão 22.0, determinou-se a curva de característica de operação do receptor (COR) e a área abaixo dela, bem como a prevalência, a sensibilidade, a especificidade, o valor preditivo positivo (VPP), o valor preditivo negativo (VPN), e acurácia, para cada teste analisado, tendo como referência padrão para o diagnóstico de DASI quando pelo menos três dos quatro testes aplicados deram positivo. Utilizou-se intervalo de confiança (IC) de 95\%.

\section{Resultados}

Foram avaliados 20 indivíduos, sendo 14 (70\%) homens, com mediana de idade de 33,5 anos (26,8-43,0 anos). Foi encontrada uma prevalência de $45 \%(n=9)$ de casos de DASI, sendo $78 \%$ (7) sintomáticos e $22 \%$ (2) assintomáticos quanto à dor (-Tabela 1).

O teste thigh thrust apresentou valor máximo de especificidade e de VPP $(1,00)$, e o teste thrust sacral, o valor máximo de sensibilidade e de VPN (1,00). Quanto à acurácia, estes mesmos testes apresentaram valores mais altos do que os demais, com superioridade para o thigh thrust (-Tabela 2).

$\mathrm{O}$ teste de força muscular da CA apresentou sensibilidade de 0,89 e especificidade de 0,82 , com VPP de 0,80 , VPN de 0,90 (-Tabela 2), e acurácia de 85\%.

A curva COR, apresentada na -Figura 1, demonstrou superioridade para o teste thigh thrust, seguido do thrust sacral e do teste da CA, sendo ratificado pela área abaixo da curva COR (-Tabela 2), que demonstrou valores para estes testes de $94 \%$, 91\% e $85 \%$, respectivamente. Os testes de compressão, distração e sintomatologia dolorosa apresentaram acurácia mais baixa $(\leq 80 \%)$. 
Tabela 2 Valores de acurácia diagnóstica dos testes clínicos para a avaliação da articulação sacroilíaca

\begin{tabular}{|l|l|l|l|l|l|l|}
\hline & Distração & Thigh thrust & Compressão & Thrust sacral & $\begin{array}{l}\text { Sintomatologia } \\
\text { de dor }\end{array}$ & $\begin{array}{l}\text { Teste da } \\
\text { cinesiologia aplicada }\end{array}$ \\
\hline Prevalência & $0,30(0,15-0,52)$ & $0,40(0,22-0,61)$ & $0,35(0,18-0,57)$ & $0,55(0,18-0,57)$ & $0,55(0,18-0,57)$ & $0,50(0,30-0,70)$ \\
\hline Sensibilidade & $0,56(0,27-0,81)$ & $0,89(0,57-0,98)$ & $0,67(0,35-0,88)$ & $1,00(0,70-1,00)$ & $0,78(0,45-0,94)$ & $0,89(0,57-0,98)$ \\
\hline Especificidade & $0,91(0,62-0,99)$ & $1,00(0,74-1,00)$ & $0,91(0,62-0,98)$ & $0,82(0,52-0,95)$ & $0,64(0,35-0,85)$ & $0,82(0,52-0,95)$ \\
\hline $\begin{array}{l}\text { Valor preditivo } \\
\text { positivo }\end{array}$ & $0,83(0,44-0,97)$ & $1,00(0,68-1,00)$ & $0,86(0,49-0,97)$ & $0,82(0,52-0,95)$ & $0,64(0,35-0,85)$ & $0,80(0,49-0,94)$ \\
\hline $\begin{array}{l}\text { Valor preditivo } \\
\text { negativo }\end{array}$ & $0,71(0,45-0,88)$ & $0,92(0,65-0,99)$ & $0,77(0,50-0,92)$ & $1,00(0,70-1,00)$ & $0,78(0,45-0,94)$ & $0,90(0,60-0,98)$ \\
\hline Acurácia & $0,75(0,53-0,88)$ & $0,95(0,76-0,99)$ & $0,80(0,58-0,92)$ & $0,90(0,70-0,97)$ & $0,70(0,48-0,85)$ & $0,85(0,64-0,95)$ \\
\hline $\begin{array}{l}\text { Área abaixo } \\
\text { da curva COR }\end{array}$ & $0,73(0,50-0,97)$ & $0,94(0,82-1,00)$ & $0,79(0,57-1,00)$ & $0,91(0,77-1,00)$ & $0,71(0,47-0,94)$ & $0,85(0,67-1,00)$ \\
\hline
\end{tabular}

Abreviatura: COR, característica de operação do receptor.

Nota: intervalos de confiança (ICs) de $95 \%$.

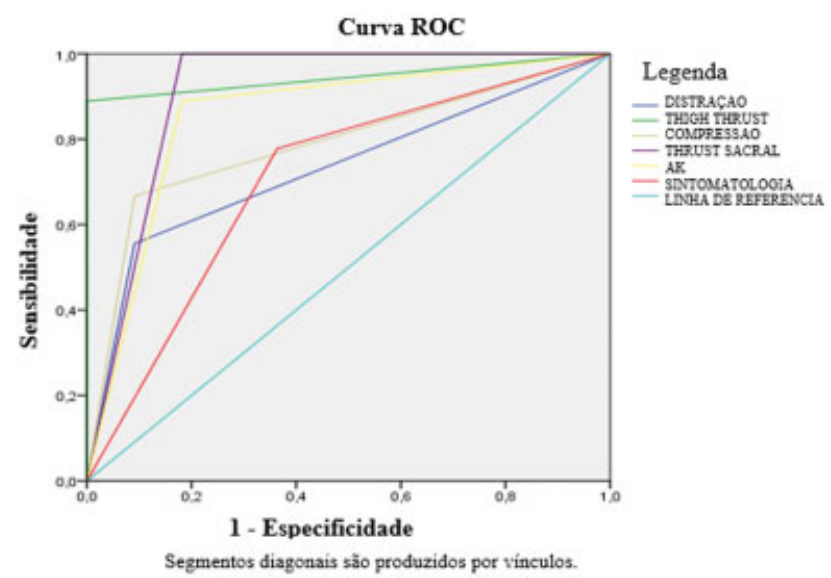

Fig. 1 Curva de característica de operação do receptor (COR) dos testes utilizados.

\section{Discussão}

A avaliação da qualidade do teste de força muscular da CA para diagnosticar DASI por meio do desafio mecânico obteve bons resultados (medidas de validade $>80 \%$ ), com destaque para a sensibilidade e o VPN.

O teste thigh thrust apresentou alto valor de sensibilidade (89\%), semelhante ao encontrado por Ramírez e Lemus, ${ }^{4}$ que foi $83,3 \%$. A especificidade encontrada nesse teste foi de $100 \%$, o que ratifica um dos achados da revisão sistemática de Stuber, ${ }^{10}$ e é compatível com o estudo de Arnbak et al, ${ }^{25}$ que encontraram um valor de $85 \%$. O estudo de Laslett et $\mathrm{al}^{8}$ verificou, em 48 pacientes que procuraram um consultório de radiologia em Nova Orleans, Estados Unidos, especializado em diagnóstico de dor na coluna, que o teste thigh thrust, quando comparado ao padrão ouro, obteve um valor de sensibilidade de $88 \%$ e VPN de $92 \%$, valores próximos aos encontrados neste estudo.

O teste thrust sacral obteve, no estudo de Laslett et $\mathrm{al}^{8}{ }^{8}$ sensibilidade de $63 \%$, especificidade de $75 \%$, VPP de $56 \%$, e VPN de $80 \%$. Na presente pesquisa, esse teste apresentou resultados expressivos, tendo alcançado os maiores escores em sensibilidade e VPN (100\% em ambos), além de resultados expressivos também de especificidade e VPP (82\% em ambos). Ramírez e Lemus, ${ }^{4}$ em seu estudo, obtiveram $100 \%$ de sensibilidade, assim como na pesquisa de Stuber, ${ }^{10}$ na qual esse teste obteve o maior valor nesse quesito.

Os quatro testes utilizados neste estudo também foram avaliados por Laslett et $\mathrm{al}^{8}$ e comparados ao padrão ouro, tendo o conjunto deles obtido valores de $78 \%$ de especificidade, $88 \%$ de sensibilidade, 67\% de VPP, e 93\% de VPN. Numa validade concorrente, quando comparados estes resultados com os obtidos com o teste da CA no presente estudo, tem-se uma superioridade em valores absolutos para a CA nos quesitos especificidade (82\%), sensibilidade (89\%), e VPP (80\%).

Um fator relevante a ser colocado a respeito da diferença, tanto do teste padrão ouro quanto dos testes ortopédicos compressivos, para o teste da AK é que estes acabam por diagnosticar uma disfunção em razão de uma sintomatologia, no caso, a dor. Além disso, os testes não especificam qual é a disfunção presente, apenas confirmam a presença de uma delas. $\mathrm{O}$ teste da CA, baseado no desafio, já direciona o terapeuta para o tratamento, uma vez que, dando resultado positivo, indica uma disfunção específica. ${ }^{15,24,25}$ No caso da articulação sacroilíaca, essas possíveis disfunções são descritas no primeiro passo da fase 2 dos procedimentos da técnica.

O teste de força muscular da CA baseia-se no princípio de verificação da resposta muscular frente ao desafio realizado, ou seja, se o músculo consegue se adaptar à mudança de pressão da força do examinador, tendo assim uma função elétrica neurológica eficaz. ${ }^{26,27}$ Waxenegge et al, $^{28}$ verificaram que o teste da CA foi uma ferramenta útil de prognóstico para os efeitos de uma terapia na qual um fármaco foi utilizado para reduzir o nível de colesterol. Cuthbert e Goodheart, $^{29}$ em uma revisão, concluíram que o teste da CA mostrou ser uma ferramenta clinicamente útil para o diagnóstico de disfunções neuromusculoesqueléticas.

Sabe-se que este estudo tem algumas limitações, sobretudo o baixo tamanho da amostra. Isso deve ter resultado em intervalos de confiança maiores e menor precisão na diferenciação da acurácia entre os testes. No entanto, este é o primeiro estudo 
que investiga a acurácia do teste de força muscular da CA para DASI com rigor metodológico no detalhamento da aplicação do teste e cegamento dos avaliadores.

\section{Conclusão}

O teste de força muscular da CA mostrou-se ser acurado para diagnosticar DASI, com acurácia semelhante à de outros testes já conhecidos, como o thigh thrust e o thrust sacral, e melhor do que os de compressão e distração. Destaca-se ainda o baixo custo e alta viabilidade clínica de uso do teste da CA, além de maior poder discriminatório quanto ao rastreio da disfunção existente. Recomenda-se a realização de outros estudos de acurácia do teste da CA na articulação sacroilíaca com amostra maior e fazendo uma comparação com o bloqueio articular por fluoroscopia.

\section{Conflito de Interesses}

Os autores declaram não haver conflito de interesses.

\section{Referências}

1 Hoy D, Bain C, Williams G, et al. A systematic review of the global prevalence of low back pain. Arthritis Rheum 2012;64(06): 2028-2037

2 van Tulder M, Becker A, Bekkering T, et al. COST B13 Working Group on Guidelines for the Management of Acute Low Back Pain in Primary Care. Chapter 3. European guidelines for the management of acute nonspecific low back pain in primary care. Eur Spine J 2006;15(Suppl 2):S169-S191

3 Strine TW, Hootman JM. US national prevalence and correlates of low back and neck pain among adults. Arthritis Rheum 2007;57 (04):656-665

4 Ramirez CR, Lemus DM. Disfunção da articulação sacro-ilíaca em jovens com dor lombar. Fisioter Mov 2010;23(03):419-428

5 Rupert MP, Lee M, Manchikanti L, Datta S, Cohen SP. Evaluation of sacroiliac joint interventions: a systematic appraisal of the literature. Pain Physician 2009;12(02):399-418

6 Hansen HC, McKenzie-Brown AM, Cohen SP, Swicegood JR, Colson JD, Manchikanti L. Sacroiliac joint interventions: a systematic review. Pain Physician 2007;10(01):165-184

7 Kennedy DJ, Engel A, Kreiner DS, Nampiaparampil D, Duszynski B, MacVicar J. Fluoroscopically Guided Diagnostic and Therapeutic Intra-Articular Sacroiliac Joint Injections: A Systematic Review. Pain Med 2015;16(08):1500-1518

8 Laslett M, Aprill CN, McDonald B, Young SB. Diagnosis of sacroiliac joint pain: validity of individual provocation tests and composites of tests. Man Ther 2005;10(03):207-218

9 Robinson HS, Brox JI, Robinson R, Bjelland E, Solem S, Telje T. The reliability of selected motion- and pain provocation tests for the sacroiliac joint. Man Ther 2007;12(01):72-79

10 Stuber KJ. Specificity, sensitivity, and predictive values of clinical tests of the sacroiliac joint: a systematic review of the literature. J Can Chiropr Assoc 2007;51(01):30-41

11 van der Wurff P, Buijs EJ, Groen GJ. A multitest regimen of pain provocation tests as an aid to reduce unnecessary minimally invasive sacroiliac joint procedures. Arch Phys Med Rehabil 2006;87(01):10-14

12 Simopoulos TT, Manchikanti L, Singh V, et al. A systematic evaluation of prevalence and diagnostic accuracy of sacroiliac joint interventions. Pain Physician 2012;15(03):E305-E344

13 Hall S, Lewith G, Brien S, Little P. A review of the literature in applied and specialised kinesiology. Forsch Komplement Med 2008;15(01):40-46

14 Gin RH, Green BN. George Goodheart, Jr., D.C., and a history of applied kinesiology. J Manipulative Physiol Ther 1997;20(05): 331-337

15 Garten H. Education: Diplomate of the International College of Applied Kinesiology. Introduction and: Systemic Disorders, General Strategies. München: German Medical Society for Applied Kinesiology; 2008

16 Schwartz SA, Utts J, Spottiswoode SJ, et al. A double-blind, randomized study to assess the validity of applied kinesiology (AK) as a diagnostic tool and as a nonlocal proximity effect. Explore (NY) 2014;10(02):99-108

17 Schmitt WH Jr, Leisman G. Correlation of applied kinesiology muscle testing findings with serum immunoglobulin levels for food allergies. Int J Neurosci 1998;96(3-4):237-244

18 Peterson KB. A preliminary inquiry into manual muscle testing response in phobic and control subjects exposed to threatening stimuli. J Manipulative Physiol Ther 1996;19(05):310-316

19 Pollard H, Bablis P, Bonello R. Can the ileocecal valve point predict low back pain using manual muscle testing? Chiropr J Aust 2006; 36(02):58-62

20 Laslett M. Evidence-based diagnosis and treatment of the painful sacroiliac joint. J Manual Manip Ther 2008;16(03):142-152

21 Eskander JP, Ripoll JG, Calixto F, et al. Value of Examination Under Fluoroscopy for the Assessment of Sacroiliac Joint Dysfunction. Pain Physician 2015;18(05):E781-E786

22 Szadek KM, van der Wurff P, van Tulder MW, Zuurmond WW, Perez RS. Diagnostic validity of criteria for sacroiliac joint pain: a systematic review. JPain 2009;10(04):354-368

23 Schmitt C, Hahn PT. A fisioterapia na síndrome do músculo piriforme: uma revisão da literatura. Rev UNINGÁ Rev. 2013;16 (01):18-21

24 Lawson A, Calderon L. Interexaminer agreement for applied kinesiology manual muscle testing. Percept Mot Skills 1997;84 (02):539-546

25 Arnbak B, Jurik AG, Jensen RK, Schiøttz-Christensen B, van der Wurff $P$, Jensen TS. The diagnostic value of three sacroiliac joint pain provocation tests for sacroiliitis identified by magnetic resonance imaging. Scand J Rheumatol 2017;46(02):130-137

26 Walter DS, Gavin DM. Applied Kinesiology. Synopsis. 2nd ed. Pueblo: Systems DC; 1988

27 Oti J. The use of manual muscle testing to assess functional integration of high-threshold versus low-threshold alpha motor neurons. In: Proceedings of the International College of Applied Kinesiology; 2004

28 Waxenegger I, Endler PC, Wulkersdorfer B, Spranger H. Individual prognosis regarding effectiveness of a therapeutic intervention using pre-therapeutic "kinesiology muscle test". ScientificWorldJournal 2007; 7:1703-1707

29 Cuthbert SC, Goodheart GJ Jr. On the reliability and validity of manual muscle testing: a literature review. Chiropr Osteopat $2007 ; 15: 4$ 